\title{
Young Children Want to See Others Get the Help They Need
}

\author{
Robert Hepach \\ Leipzig University, Max Planck Institute for Evolutionary \\ Anthropology
}

\author{
Amrisha Vaish, and Tobias Grossmann \\ University of Virginia
}

\author{
Michael Tomasello \\ Duke University, Max Planck Institute for Evolutionary Anthropology
}

\begin{abstract}
Children's instrumental helping has sometimes been interpreted as a desire to complete action sequences or to restore the physical order of things. Two-year-old children $(n=51)$ selectively retrieved for an adult the object he needed rather than one he did not (but which equally served to restore the previous order of things), and those with greater internal arousal (i.e., pupil dilation) were faster to help. In a second experiment $(n=64)$, children's arousal increased when they witnessed an adult respond inappropriately to another adult's need. This was not the case in a nonsocial control condition. These findings suggest that children's helping is not aimed at restoring the order of things but rather at seeing another person's need fulfilled.
\end{abstract}

When young children see another person in need, they often respond with prosocial behavior such as comforting (Eisenberg \& Miller, 1987; Zahn-Waxler, Radke-Yarrow, Wagner, \& Chapman, 1992), informing (Liszkowski, Carpenter, \& Tomasello, 2008), sharing resources (Brownell, Svetlova, \& Nichols, 2009; Svetlova, Nichols, \& Brownell, 2010), and more generally aiding others in completing their goaldirected instrumental actions (Warneken \& Tomasello, 2006, 2007). Given that these behaviors benefit others, it is tempting to conclude that children are motivated by the prosocial desire to enhance others' welfare. However, in order to conclude that early prosocial behavior is the result of truly prosocial motives, alternative explanations first need to be ruled out (Hepach, Vaish, \& Tomasello, 2013a).

In a recent study, Hepach, Vaish, and Tomasello (2012) addressed one such alternative explanation of instrumental helping behavior, namely, that

We thank all the parents and children who participated in our studies and Jana Jurkat and Elvira Portner for their help with running the study. The house apparatus was built by Henrik Röthel and decorated by Isabelle de Gaillande-Mustoe, Alina Schönfuß, Cleo Meinicke, and Parissa Chokrai. We are grateful to Jessica Bürgel and Julia Ulber for their help with coding and reliability as well as to Roger Mundry for his advice on statistics. In addition, we thank Robert Schettler for his IT support, Ronny Barr for his support with Adobe, and Karla Schmölling for her help with the videos. This research was partially supported by a grant from the DFG (German Research Foundation) Excellence Cluster 302: Languages of Emotions.

Correspondence concerning this article should be addressed to Robert Hepach, Department of Research Methods in Early Child Development, Faculty of Education, Leipzig University, 04109 Leipzig. Electronic mail may be sent to robert.hepach@unileipzig.de. children may be motivated to seek out recognition for their actions rather than caring for others' wellbeing. The authors found that 2-year-old children's internal arousal decreased not only when they themselves returned an object that an adult had accidentally dropped but also when they could not provide help but someone else did so, whereas it remained high when the adult was not helped at all. Furthermore, the greater children's internal arousal after witnessing the situations, the quicker they were to help the adult (Hepach et al., 2013a). This suggests that children were not merely motivated to "get credit" for helping but were genuinely concerned to see the person in need be helped.

Nonetheless, in all documented cases of children's instrumental helping, one could hypothesize that the child is motivated by the social exchange (e.g., Lennon \& Eisenberg, 1987) or simply attempting to complete a sequence and restore the previous order of things regardless of the person's actual need, for instance, that objects that have been dropped have to be picked up and put back where they were. This latter alternative interpretation also holds true when considering the control conditions of previous studies in which children do not return an object if the adult explicitly discards it by intentionally throwing it on the floor, that is, when there is no incomplete sequence (Dunfield, O'Connell, Kuhlmeier, \& Kelley, 2011;

(C) 2016 The Authors

Child Development (C) 2016 Society for Research in Child Development, Inc. All rights reserved. 0009-3920/2016/8706-0005

DOI: $10.1111 /$ cdev.12633 
Warneken \& Tomasello, 2006). Indeed, children's goal-directed helping (as it also occurs in other nonhuman primates such as chimpanzees) could be driven not by a concern for the individual in need but rather out of a desire to fulfill incomplete action sequences (Köhler, 1925). Given the parallels between young children's and nonhuman primates' instrumental helping behavior, the alternative explanations for chimpanzee helping may equally well apply to that of young children (Warneken, Hare, Melis, Hanus, \& Tomasello, 2007). Thus, what is commonly referred to as helping may actually be driven by an underlying nonsocial motive to see the order of things restored.

We conducted two experiments to address this alternative explanation of young children's instrumental helping behavior. We did so by using an experimental paradigm recently developed by Hepach et al. (2012) to study the motives underlying children's behavior. The authors measured changes in children's internal arousal via their pupil dilation, an established measure in psychophysiology (Beatty \& Lucero-Wagoner, 2000; Laeng, Sirois, \& Gredebäck, 2012) indicative of changes in arousal of the autonomous nervous system (Loewenfeld, 1958). In the present work, in the first experiment, we investigated whether children would help an adult by handing him the object he needed rather than one he did not (but which equally served to restore the previous order of things) and whether children's internal arousal, that is, pupil dilation, was related to their willingness to help. In the second experiment, we investigated whether children's internal arousal varied as a function of whether an actor's need was fulfilled by the response of another person (social condition) and included a nonsocial control condition in which the movement of objects was identical to the social condition but no persons were present and thus there was no unfulfilled need. The two experiments were conducted during the same laboratory visit in a fixed order for all children. For practical purposes, Experiment 1 was run after Experiment 2, but we present Experiment 1 first for conceptual reasons and to increase the clarity and coherence of the article for the reader. Experiment 1 serves the function of validating pupil dilation as a measure of motivation and is therefore foundational to and facilitates the interpretation of Experiment 2 (see Supporting Information for further details).

\section{Experiment 1}

The aim of Experiment 1 was to validate the use of pupil dilation as a measure of internal arousal to assess children's motivation to help (see also Hepach et al., 2012, 2013a) and to investigate whether children would be sensitive to another's need if they could provide help themselves. Children were presented with a live situation in which an adult dropped multiple objects that he could not reach, and thus he could not finish his task. Half the objects were relevant, the other half irrelevant to his task. Children's pupil dilation before they got to help the adult as well as their latency to pick up the first object were measured. In addition, we coded for the type of object (relevant or irrelevant) that children picked up first. We hypothesized that children would be more likely to pick up the relevant over the irrelevant objects and that the level of children's arousal would be correlated with their latency to help (see also Hepach et al., 2013a). Additional data and analyses are provided in the Supporting Information.

\section{Method}

\section{Participants}

Participants were 2-year-old children recruited from the department's database (median age $=2$ years, 1 month, 2 days; age range $=2 ; 0 ; 0$ 2;2;0). Data collection took place at the Max Planck Institute for Evolutionary Anthropology in the midsized city of Leipzig, Germany, between May 2012 and April 2013. Children came from mixed socioeconomic backgrounds and all children were native German speakers. A total of 51 children ( 25 boys) were included in the final analyses. Six additional children were tested but excluded from the final sample because of fussiness.

\section{Materials and Design}

Children were presented with a wooden house apparatus $(1.35 \mathrm{~m}$ wide $\times 1.64 \mathrm{~m}$ high $\times 0.20 \mathrm{~m}$ deep). A window $(64 \times 68 \mathrm{~cm} ; 46 \mathrm{~cm}$ above the ground) held the computer monitor (24-in.; $52 \times 32 \mathrm{~cm})$ at a resolution of $1920 \times 1080$ pixels as well as the eye tracking unit (Tobii model X120; Tobii Technology, Stockholm, Sweden) with a sampling frequency of $60 \mathrm{~Hz}$ and bright pupil capture setting (see also Jackson \& Sirois, 2009) that was used to measure children's eye movements and pupil diameter (see Figure 1). Participants' eyes were calibrated using a 5-point calibration procedure (see also Gredebäck, Johnson, \& von Hofsten, 2010). Stimulus presentation and data recording was carried out using Tobii Studio (Version 3.1.3; Tobii Technology). 


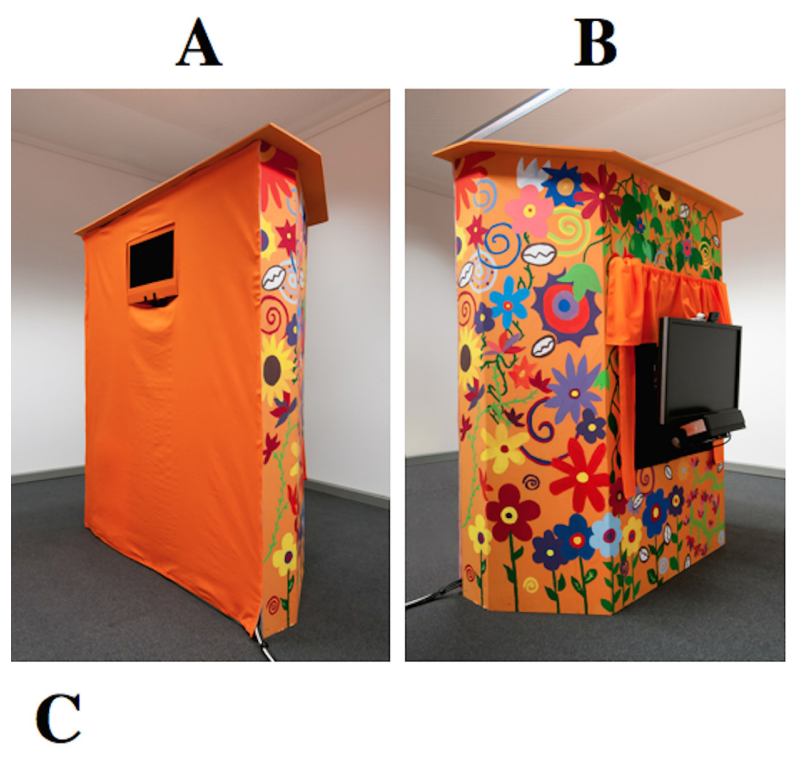

Sequence of Events During Test Trial

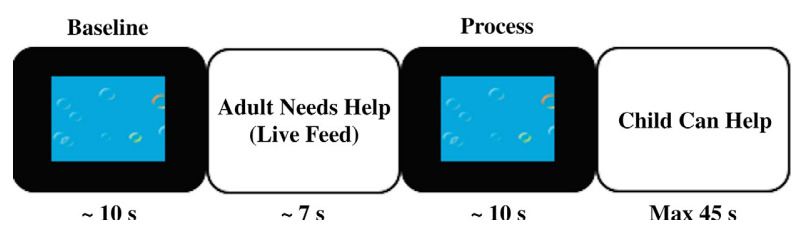

Figure 1. Apparatus used in Experiment 1 (A and B) and stimulus sequence during the test trial $(\mathrm{C})$. Between the two neutral clips, E1 continued his action and after $2 \mathrm{~s}$ "accidentally" dropped 12 objects from the center of the table. He expressed confusion, pretending to not know where the objects went (5s).

Each child saw two test situations adapted from Warneken and Tomasello (2006). In one situation, Experimenter 1 (E1) sat behind a table and hung up pieces of cloth with clothes pegs. In another situation, E1 drew a picture with a set of crayons. Placed at the center of the table were 12 objects, 6 clothes pegs and 6 crayons. At a predetermined point, E1 "accidentally" dropped all 12 objects on the floor. A separate preference test with nine participants (from a different group of children) established that children at this age do not prefer crayons over clothes pegs. To measure pupil dilation, we presented neutral clips (see Supporting Information and Figure 1) at the beginning of each trial (baseline measure) and before children got the chance to pick up objects for the adult (process measure). The order of the two test situations and the color of the neutral clips (blue or purple) were counterbalanced across children.

To obtain a wider range of values for children's latency to help the adult we used a distracter toy (a baby swimming pool filled with balls) such that children had to decide whether to first help the adult or to engage in a fun activity themselves (see also Warneken \& Tomasello, 2012).

\section{Procedure}

The experiment was run by three experimenters and was divided into three phases: warm-up, familiarization, and test. During the warm-up phase E1 played with the child while Experimenter 2 (E2) explained the study to the parent. Parents gave informed consent before the study began and were instructed to close their eyes while sitting in front of the monitor during the test phase and to not provide their children with any cues. E2 introduced the child to the house apparatus. This was the beginning of the familiarization phase (adapted from Hepach et al., 2012; see Supporting Information for details). Children followed an exchange of objects while sitting in front of the computer monitor thus establishing a contingency between E1's and the child's side. Furthermore, children were encouraged to move to the inside and back to the outside of the house. After the familiarization, E3 entered the room and brought with her the distracter toy and placed it at approximately $2.25 \mathrm{~m}$ away from E1. E2 and the child examined the distracter toy.

The test phase began with E1 either hanging up pieces of cloth or drawing a picture. After $40 \mathrm{~s}$ E2, the parent, and the child moved back in front of the house and the child sat on the parent's lap (see Figure 1 for a sequence of the test stimuli). After children saw E1 needing help and after the process measure of pupil dilation was taken, parents carried their child to the inside of the house to a distance of approximately $2.5 \mathrm{~m}$ from E1 and approximately $2 \mathrm{~m}$ from the distracter toy. Children were allowed to move around freely. E1 showed a neutral facial expression and then systematically increased the cues expressing his need for help: (a) alternating gaze between the objects and the child, (b) after $7 \mathrm{~s}$ (from the start) reaching for the object and looking at the child, (c) after $15 \mathrm{~s}$ saying "Oh look child's name [. . .] Look down there. [. . .] I cannot get to it," (d) after $30 \mathrm{~s}$ directly addressing the child to ask for help (see Svetlova et al., 2010 for a similar cue procedure). E1 continued to provide the above cues until children had picked up at least six objects (whether relevant or irrelevant), after which he began sorting the objects on the table. If a child offered him the toy, the adult took it regardless of whether or not it was a relevant or an irrelevant one. E1 did not provide the child with any verbal 
feedback such as "Thank you." The trial ended either after children had picked up all the objects or if they did not pick up any objects, then after $45 \mathrm{~s}$ had passed. Once children started picking up objects, the trial did not end after $45 \mathrm{~s}$ but ended when they had picked up all the objects or if they disengaged from the activity and moved away from the table.

\section{Data Analysis}

Pupil dilation. For pupil dilation analyses, only data from the neutral clips were used. As an indicator of the extent of pupil dilation, we identified the average absolute minimum in pupil dilation for each time point in each test trial (see also Steinhauer, Condray, \& Kasparek, 2000 for a similar analysis). The idea is that an increase in internal arousal will lead to an inhibited pupillary light reflex, therefore leaving the pupils more dilated. More specifically, because the neutral clips consisted of two presentations of the colorful images, two pupillary light reflexes (PLR) were elicited, and therefore two absolute minima were obtained. The average of those two minima within a neutral sequence was used for further analyses Measuring pupil dilation in this way greatly reduces noise to allow for single-trial analyses. The identification of PLRs was standardized using an algorithm written in R (version 2.15.1, R Core Team, 2012; see Supporting Information for details). On a given trial, a participant provided data for two time points: the baseline measure and the process measure.

Behavioral data. We coded how many objects children picked up and how long it took them to pick up the first object from the moment when parents carried children to the marked spot inside the house until the first object was either placed on the table or in E1's hand. Children who did not help were assigned the maximum latency of $120 \mathrm{~s}$, which was approximately the duration of a trial until children picked up all the objects. Furthermore, we were also interested in whether children gave a relevant or an irrelevant object to E1 first. For this, we identified the type of object children handed to E1 first. Furthermore, we looked at the ratio of relevant objects among the first six objects children picked up in order to have a more robust measure of their preference for the type of object. The first coder, not blind to hypotheses, coded all the trials. A second coder, blind to hypotheses, looked at a random sample of 30\% of the data and reliability was high for the total number of objects, $\kappa($ Cohen $)=.95$, for the type of object, $\kappa($ Cohen $)=.89$, and for the ratio of correct objects, $\kappa($ Cohen $)=.91$. In addition, agreement for the latencies was perfect (Intraclass Correlation Coefficient $($ ICC $)=1$ ). To assess children's initial, unbiased responses, we focused our analyses on the first test trial. Additional data and analyses are provided in the Supporting Information.

\section{Results}

The majority of children (33 of the $51 ; \sim 65 \%$ ) picked up an object. The average number of objects picked up by a child was 9 (range $=1-12$ ). Furthermore, 23 of the $33(\sim 70 \%)$ children picked up a relevant object first, binomial test (two-tailed) $p=.035$. In addition, the average ratio of relevant objects among the first six objects $(M=0.6, S D=.28)$ was significantly above chance $(50 \%), t(32)=2.1$, $p=.04$. Of the 18 children who did not pick up an object, two stood close to E1 but did not intervene, seven went to the distracter game, and nine children stayed with their parent. To investigate the relation between children's pupil dilation and their motivation to help, we calculated correlations between both children's baseline measure and process measure of pupil dilation and children's latency to pick up the first object. In case a participant did not pick up an object at all, the maximum latency value of 120 s was used (see Data Analysis). Children started picking up the first object after $25.58 \mathrm{~s} \quad(S D=16.3 \mathrm{~s})$, and those children who showed greater pupil dilation in the process measure were faster to pick up the first object for the adult, Spearman's rho $(\rho ; n=45)=-.31 ; p=.04$. Importantly, there was no association between children's baseline level of pupil dilation and their latency to pick up the first object, $\rho(n=47)=-.13$; $p=.37$. In addition, there was no difference in children's process measure of pupil dilation between children who picked up the irrelevant object first $(M=3.792, S D=.69)$ and those who picked up the relevant object first $(M=3.788, S D=.55), \quad t$ $(29)=0.02, p=.98$.

Further analyses revealed no effect of the type of situation (crayons vs. clothes pegs) or participants' gender on any of the dependent measures and no difference in children's process measure of pupil dilation or its relation to the latency to help depending on what type of object children picked up first $(p s>.33)$. Moreover, with regard to the main findings, we obtained the same pattern of results on the second test trial though the effects did not reach statistical significance (see Supporting Information for details). 


\section{Discussion}

The aim of Experiment 1 was to investigate whether children would be sensitive to an adult's need by preferentially providing him with the objects relevant to his task. In addition, we were interested in whether there was an association between children's internal arousal, that is, pupil dilation, and their motivation to help. The main finding was that children were sensitive to the adult's need both in their initial choice and when considering the ratio of the first half of objects picked up. Overall, they preferred to pick up objects relevant to the adult's task to those that were irrelevant. This finding is particularly striking given that children had to identify the six relevant objects among a total of 12 objects that had dropped. This is conceivably more demanding than the instrumental helping tasks used in previous studies, in which only one object dropped and hence the only decision children had to make was whether or not to pick it up (Dunfield et al., 2011; Warneken, 2013; Warneken \& Tomasello, 2006). Indeed, the rate of helping in the present study is lower than the rate in studies in which only one object was dropped but is comparable to the overall helping rate in other studies in which children had to pick up multiple objects to help an adult (e.g., Over \& Carpenter, 2009) or in which children were also presented with a distracter toy (Warneken \& Tomasello, 2012). In addition, children had no prior experience with either type of object. Furthermore, children's pupil dilation was linked to how quick they were to pick up the first object for the adult. In other words, the more aroused children were before upon seeing the adult needing help, the faster they picked up an object for the adult. Children's process measure of pupil dilation was not related to the type of object they picked up first, thereby suggesting that this change in internal arousal reflects a general motivation to help rather than the specific order in which children want to pick up the objects to fulfill others' needs.

One question that arises is why children did not only pick up the relevant objects. It could be argued that if children wanted to help the adult, then they should only have picked up the relevant objects and not the irrelevant ones. A possible explanation is that children found it difficult to inhibit an action once they had started it. That is, once the behavior of picking up objects was set in motion, children continued to do so and the initial motive to pick up a relevant object for the adult transferred to a general motive to pick up all the objects on the floor. In fact, children's initial motive may simply have been to help the adult pick up all the objects and thus help him clean up. Nevertheless, the important finding is that in doing so children were sensitive to the adult's need by preferentially handing him the relevant object first.

In sum, children were sensitive to the adult's need by selectively picking up objects relevant to his task. The question is whether they did so to see an action sequence completed rather than responding to the adult's need. This question was addressed in a second experiment to investigate whether children's internal arousal is indeed triggered by another person's unfulfilled need rather than merely by the physical aspects of the situation.

\section{Experiment 2}

The aim of Experiment 2 was to investigate whether children's arousal is triggered by a social motive to see others being helped appropriately. If children are sensitive to others' needs, we predicted that (a) children would anticipate the appropriate resolution, (b) children's pupil dilation would increase to the need situation, and (c) children's pupil dilation would decrease only if the other's need was appropriately fulfilled. We further included a nonsocial control condition to rule out that such effects were driven by the physical properties of the situation.

\section{Method}

\section{Participants}

Participants were 2-year-old children recruited from the department's database (median age $=2$ years, 1 month, 2 days; age range $=2 ; 0 ; 0$ 2;1;28). Data collection took place at the Max Planck Institute for Evolutionary Anthropology in the midsized city of Leipzig, Germany, between May 2012 and April 2013. Children came from mixed socioeconomic backgrounds, and all children were native German speakers. A total of 64 (32 male) children participated and were included in the final analyses. The total number of participants consisted of 51 children who also participated in Experiment 1 plus 13 children from the same overall population who provided data only for Experiment 2 (see Supporting Information for details). Additional children were tested but excluded from the final sample because of technical failure $(n=2)$, experimenter/ parent error $(n=2)$, or fussiness $(n=2)$. 


\section{Materials and Design}

Two types of videos were used in the experiment: action clips portrayed either an adult stacking objects (social condition) or objects being stacked without any person present (nonsocial condition). In addition, based on prior work (Hepach et al., 2012), neutral clips showing colorful bubbles on a colored background were presented at specific time points to assess changes in participants' pupil dilation (see also Experiment 1).

In the social condition, one adult (actor) sat behind a table at the center of the screen and another adult (observer) sat on a chair on the righthand side of the screen. Five objects of similar kind (e.g., cans) were arranged on the right side of the table, and two objects were positioned in the center: one relevant object (e.g., another can) and one irrelevant object (e.g., a shoe). Subsequently, the actor started moving three objects, one by one, from the right to the left side of the table while the observer watched. Next, the actor moved two more objects from the right side to the left side and stacked them on top of the previous three. The observer turned away from the scene pretending to read. When adult 1 put the fifth can on top of the other four, the two objects in the center of the table "accidentally" dropped to the floor and rolled to the center front of the scene. The animations of the objects dropping to the floor were edited into the movies using Adobe Premiere Pro to keep the movements identical in the social and nonsocial conditions (CS 5, version 5.0.3). Adult 1 expressed confusion and, upon seeing the two objects lying on the floor, reached ambiguously between the two objects while displaying an overall neutral expression. After looking at the scene for approximately $5 \mathrm{~s}$ the observer got up, picked up either the object matching the other five (relevant condition; e.g., the can was returned in the can-stacking situation) or the nonmatching object (irrelevant condition; e.g., the shoe was returned in the can-stacking situation), and handed it to the actor. The actor took the object, looked at it, and moved back to an upright position behind the table. In the nonsocial condition, one of the objects "flew back" on the table.

The timing of events within a segment, for example, when the objects dropped, as well as the overall duration of segments was the same for all versions of the clips. In addition, the use of computer animation software allowed for the movement of objects to be kept identical between the social and nonsocial versions of the clips. Furthermore, the position of the relevant and irrelevant objects on the table, and subsequently the side to which they fell on the floor, was counterbalanced. In order to measure pupil dilation on the same stimulus at specific time points during the presentation of the action clips, the neutral clips were presented while the actor was stacking (baseline measure), after he reached for the objects (process measure) and after the observer gave one object to the actor (post measure). The total duration for a test trial was approximately $1 \mathrm{~min} 40 \mathrm{~s}$.

Each participant watched a different situation on each test trial (order counterbalanced), one in which cans were stacked and a shoe was the irrelevant object and another version in which shoes were stacked and a can was the irrelevant object. Children were either tested in the social or the nonsocial condition (between subjects factor). All children were presented with two test trials, one in which the relevant object (relevant condition; e.g., a can was returned in the can-stacking situation) and another in which the irrelevant object (irrelevant condition; e.g., a can was returned in the shoestacking situation) returned (within subjects factor, order counterbalanced).

\section{Procedure}

The experiment was run by two female experimenters (same experimenters as in Experiment 1) and included a warm-up, calibration, and test phase. During the warm-up, E1 explained the study to parents, while E2 played with the child. Parents gave informed consent before the commencement of the experiment and were asked to keep their eyes closed during the study and to not provide their child with any cues. E2 left the study room and E1 directed the child's attention to a computer screen. Children were seated on their parents' lap. After the calibration phase, E1 moved behind the parent, out of the child's view, and the test phase began. E2 ran the stimulus presentation procedure from a separate room (further details are provided in the Supporting Information).

\section{Data Analysis}

Looking time. For each participant, the raw gaze data were corrected based on a procedure developed by Frank, Vul, and Saxe (2012). Each recording file was exported from Tobii Studio to Matlab (version 7.12, R2012a) and a series of Matlab routines written by Frank et al. (2012) were adapted for the present purposes to post hoc correct participants' point of gaze (see Supporting Information 
for more details.). For two participants in the social condition, no such calibration correction could be performed because of poor attention and data acquisition during the video clip, and those subjects were excluded from further looking time analyses. For the remaining subjects, only samples belonging to a fixation were used. Within Tobii Studio, fixations were defined with a window length of $20 \mathrm{~ms}$ and a velocity threshold of $30 \%$ s. Furthermore, adjacent fixations were merged with a maximum time of $75 \mathrm{~ms}$ and a maximum angle of $0.5^{\circ}$ between fixations. In addition, the crucial time window for analyzing looking behavior were the $13 \mathrm{~s}$ after the adult initiated reaching for the two objects on the floor (or the equivalent $13 \mathrm{~s}$ in the nonsocial condition) until the end of that video sequence. For each participant, we determined how much time was spent looking at both object regions of interest (width and height $=195$ px; see Figure 2). For statistical analyses, we calculated the proportion of time children looked at the correct object, that is, time correct object/(time correct object + time incorrect object). As a control variable, we included the time (in collected samples) children watched the stacking action until the adult reached for the objects (or the equivalent time point in the nonsocial control condition). On the first test trial seven participants did not look at the objects in the specified time window (six in the social and one in the nonsocial condition).

Pupil dilation. The procedure for analyzing the data followed that of Experiment 1. On a given trial, a participant provided data for the neutral clips at three time points: the baseline measure, the process measure, and the postmeasure. Within each trial, data from the process and postmeasure were baseline corrected, for example, in the case of the process measure, the difference of the average of the two PLRs of the process measure and baseline measure was divided by the average baseline measure. Therefore, the actual data points used for further analyses were the baseline-corrected values for process measure and postmeasure. In summary, each participant could provide both process measure and postmeasure for each of the two test trials, that is, four data points in total.

Similar to Experiment 1, we focused our analyses on the first test trial (see Supporting Information for additional data analyses). Consistent with our hypotheses, we carried our three separate statistical analyses focusing on (a) children's looking behavior after the objects had dropped, (b) the increase in pupil dilation following the accident, and (c) the change in pupil dilation depending on how the

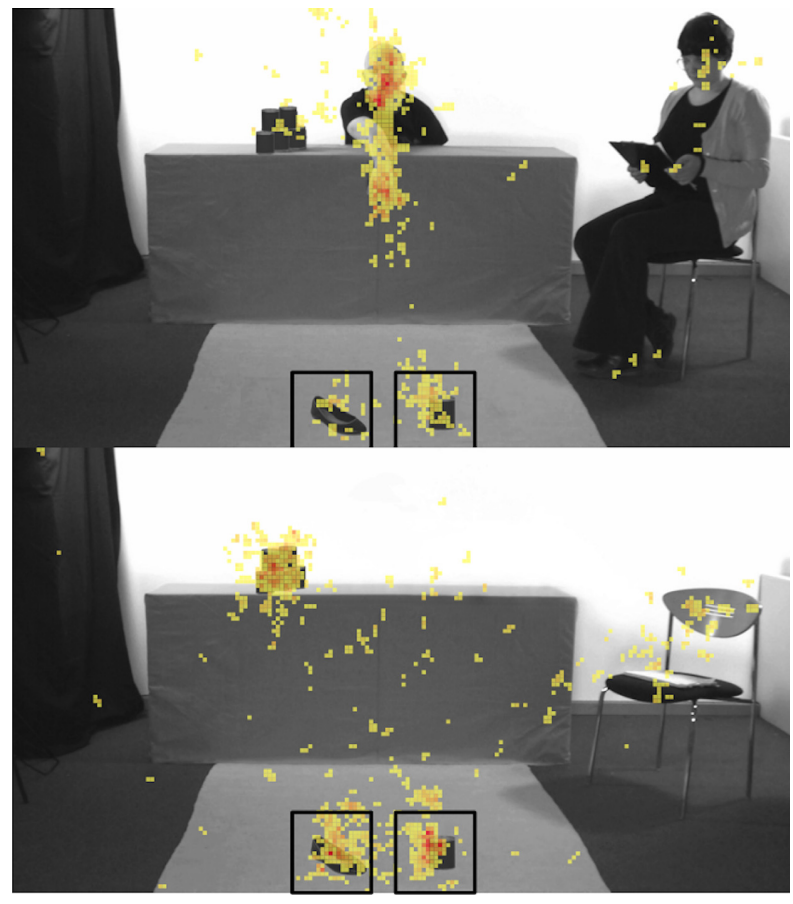

Figure 2. Still frame from the moment when the adult was reaching for the objects. The regions of interest for both objects are marked with rectangular shapes. The gaze behavior of participants on the first trial with the relevant object being on the righthand side is plotted on top of the images to illustrate the focal points of attention. Greater visual focus is represented through red coloring (darker coloring in print). The top panel shows the summary for the social condition and the bottom panel shows the summary for the nonsocial condition.

situation was resolved (i.e., with the relevant or irrelevant object). For analyses (a) and (b), we ran analyses of variance and the independent factors were condition (social vs. nonsocial) and gender. For (c), we ran analyses of covariance including the type of object returned as an independent factor as well as children's process measure of pupil dilation as a covariate and gender. Details regarding the checks of statistical model assumptions are provided in the Supporting Information.

\section{Results}

\section{Looking Time}

The proportion of time children spent looking at the relevant object after the objects dropped and before the resolution of the situation varied significantly between the social and nonsocial condition, $F$ $(1,53)=11.68, p=.001$. Children looked significantly longer at the relevant compared to the irrelevant object in the social $(n=26 ; M=0.76, S D=.27)$ but not in the nonsocial condition $(n=31 ; M=0.53$, 
$S D=.24)$, Mann-Whitney $U=622.5, p=.0003$. Furthermore, children's longer looking at the relevant object was significantly above chance, that is, more than $50 \%$ of the time, in the social, Wilcoxon exact $T=315, n$ (without ties) $=26, p=.0001$, but not in the nonsocial condition, $T=288, n=31, p=.44$ (see Figures 2 and $3 \mathrm{~A}$ ). There was no statistical effect of gender $(p=.99)$ or the time children spent looking at the stacking action $(p=.83)$. Overall, children showed anticipatory looking toward the relevant object in the social but not in the nonsocial control condition. The same pattern of results and a statistically significant effect of condition emerged when considering both test trials (see Supporting Information for details).

\section{Pupil Dilation}

The initial change in children's pupil dilation differed between the social and the nonsocial conditions, $F(1,61)=4.01, p=.0497$ (see Figure 3B). Children's pupil dilation increased more from baseline to process when watching the objects drop in the social $\left(M_{\text {so- }}\right.$ cial $=0.07$, SDsocial $=.07)$ compared to the nonsocial condition $\left(M_{\text {nonsocial }}=0.04, S D_{\text {nonsocial }}=.04\right), T=363$, $n=32, p=.046$. In addition, girls $\left(M_{\text {girls }}=0.07\right.$, $\left.S D_{\text {girls }}=.06\right)$ showed more increase in pupil dilation than boys $\left(M_{\text {boys }}=0.04, \quad S D_{\text {boys }}=.05\right)$, $\beta=-.03 \pm .01, t(59)=-2.39, p=.02$. Moreover, the same pattern of results was obtained when considering both test trials, though no statistically significant
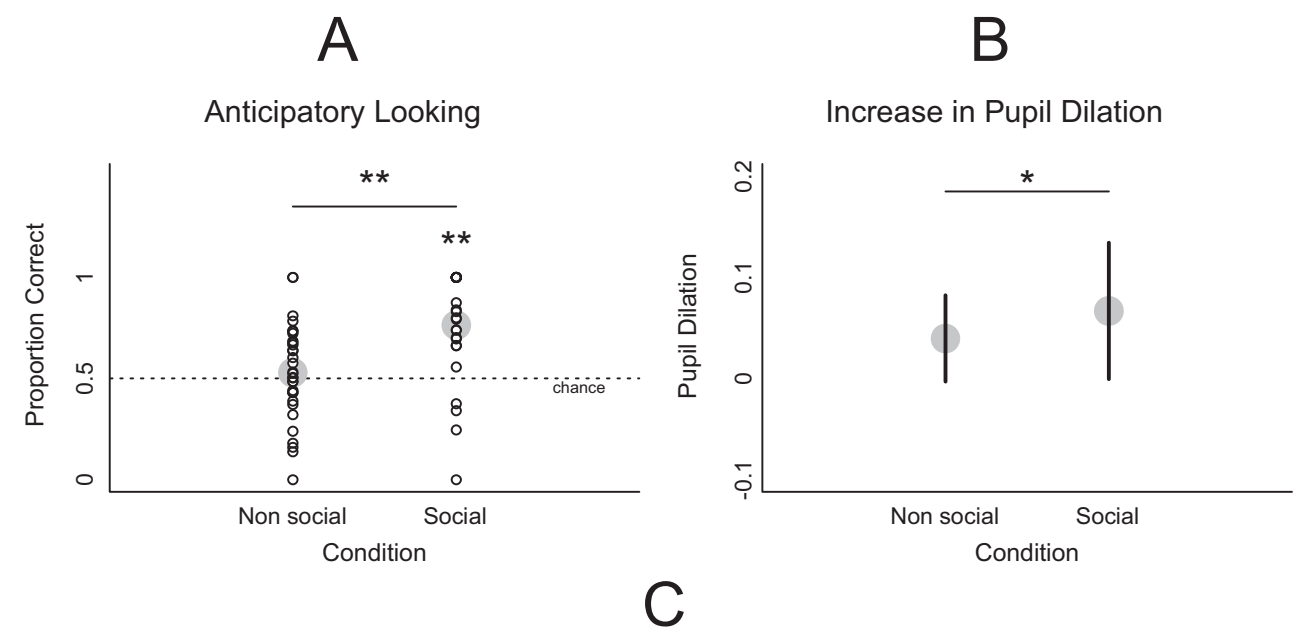

Post change in Pupil Dilation
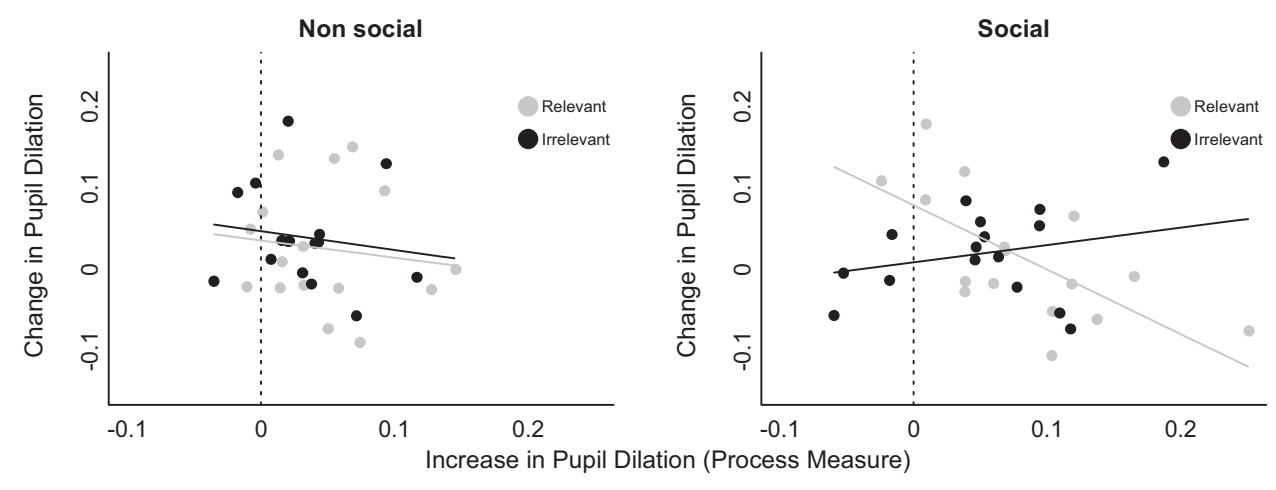

Figure 3. Data illustration of children's anticipatory looking and changes in pupil dilation on the first test trial. (A) For each participant the proportion of time looked at the correct object (divided by the time looked at both objects) after the objects dropped is plotted for both conditions. The gray circles represent the means. ${ }^{* *} p<.01$. (B) The increase in children's pupil dilation before the resolution of the situation is plotted for both conditions. The means are illustrated by gray circles. The standard deviation is indicated by the black lines. $*_{p}<.05$. (C) Children's change in pupil dilation after the resolution of the situation depending on the initial increase in arousal. The left panel shows the data for the nonsocial condition. The solid lines represent the model lines. The right panel shows the data for the social condition with the model lines. Only in the social condition, when the irrelevant object was returned, did children show a pattern different from values regressing to the mean, that is, only in this condition did large and small values in the premeasure align with large and small values in the postchange measure, respectively. 
effect of condition emerged (see Supporting Information for details).

To investigate changes in children's pupil dilation in response to the resolution of the situation we ran two separate analyses, one for the social and the other for the nonsocial condition. There was a significant interaction between children's initial increase in pupil dilation and object relevancy in the social, $F(1,27)=9.62, p=.004$, but not in the nonsocial condition, $F(1,27)=0.001, p=.98$. That is, the more children's internal arousal increased by seeing a person needing help, the more they remained aroused only if the irrelevant object was returned $(\beta=.01)$ and the more it decreased again if the relevant object was returned $(\beta=-.05)$. There was no statistical effect of gender $(p=.24)$. In the nonsocial condition, the initial arousal decreased again regardless of whether the irrelevant $(\beta=-.01)$ or the relevant $(\beta=-.009)$ object was returned (see Figure 3C). There was no statistical effect of gender $(p=.44)$. Overall, the same pattern of results reached statistical significance when incorporating both test trials. For all dependent measures, additional analyses revealed no effect of type of situation (stacking cans vs. stacking shoes) or of the location of the relevant object (left or right; see Supporting Information for details).

\section{Discussion}

The aim of Experiment 2 was to investigate whether children's internal arousal is triggered by a social motive to see others being helped appropriately. In accordance with our predictions, we found that (a) children anticipate the appropriate solution of another's situation, (b) children's pupil dilation increases to the need situation, and (c) children's pupil dilation decreases only if the other's need was appropriately fulfilled (or when it did not matter, as in the nonsocial control). In other words, children's internal arousal remained high if an adult needing help was given an object that did not adequately fulfill his need even though the physical order of things was restored in part and the adult stopped reaching. We included a nonsocial control condition and found that the effects on anticipatory looking and pupil dilation were not driven purely by the physical properties of the situation. Together, these results suggest that children's internal arousal increases as a consequence of witnessing a person needing help, and it remains high if appropriate help is not provided. Children are sensitive to others' needs and expect others to be helped appropriately.
The fact that children's pupil dilation increased more in the social compared to the nonsocial condition does not mean that children did not respond to seeing objects drop in the nonsocial condition, because in that case children's pupil dilation also increased (see Figure 3B), possibly as a function of the complexity of the situation (see also Jackson \& Sirois, 2009). Our results in the social condition are consistent with previous work on infants' understanding of social interaction showing that infants show more pupil dilation as a consequence of seeing incongruent displays of emotional faces and actions (Hepach \& Westermann, 2013) and unusual social interactions (Gredebäck \& Melinder, 2010). In the present study, seeing an adult not providing the appropriate help could be seen as an unusual interaction or a resolution that is incongruent with what the adult needs. The additional effect that girls showed greater increase in pupil dilation than boys after witnessing the problem could suggest that girls became more involved than boys (see also Fabes, Eisenberg, \& Eisenbud, 1993). However, we found no gender effect on children's pupil dilation after they saw the situation being resolved, suggesting that boys and girls alike were sensitive to seeing the adult's need fulfilled appropriately.

\section{General Discussion}

The main finding of the present experiments is that children want to see others get the help they need. In a situation where 2-year-old children themselves get the chance to help an adult and have to discriminate relevant from irrelevant objects, they selectively hand the adult the objects he needs (Experiment 1). Although the task was conceivably demanding, given that multiple objects dropped, children nevertheless preferably chose to help by handing over the relevant objects first. During those situations children's own level of internal arousal related to how quick they were to pick up the first object for an adult who could not reach it himself (as also reported by Hepach et al., 2013a). Furthermore, when children are observers of a situation in which an adult needs help, they expect to see the relevant solution and their own internal arousal increases and remains high if the adult does not get the relevant object he needs to finish a task (Experiment 2).

These findings contribute to the results from previous studies on children's prosocial behavior as they clarify the nature of young children's motivation to fulfill others' instrumental goals. Although 
prior work has shown that young children can be made aware of other's needs (e.g., Brownell et al., 2009; Svetlova et al., 2010; Warneken, 2013), the present experiments show that children at the age of 2 spontaneously prefer to help in ways that are appropriate to another person's unfulfilled need. The motivation is not only intrinsic (Warneken \& Tomasello, 2008) but appears to be specifically triggered by the social component of the situation given that the nonsocial condition in the present study failed to elicit a similar degree of involvement and attention.

Furthermore, the correlation we found in Experiment 1 replicates a previous finding that 2-year-old children's arousal state upon seeing a person need help correlates with their latency to help that person (see Hepach et al., 2013a). Importantly, our measure of children's baseline level of pupil dilation did not correlate with their latency to help, showing that the main source for the correlation was not simply children's baseline state of arousal but specifically their arousal in response to seeing the person need help. This further validates the use of physiological measures such as pupil dilation to assess children's motivation to help (see also Fabes et al., 1993; Hastings, Zahn-Waxler, \& McShane, 2006). It is important to note that the results from Experiment 1 suggest that the degree to which children's internal arousal changes to others' in need reflects a more general motivation to help rather than reflecting the specific in which children want to help those in need.

Whereas previous studies had investigated when and how often young children help others (e.g., Dunfield et al., 2011; Svetlova et al., 2010; Warneken \& Tomasello, 2006, 2007, 2013), the present work further specifies the motivational mechanism underlying early helping behavior. First, pupil dilation is not a measure of a specific emotion such as sympathy or a particular thought but rather a symptom of psychological engagement. At any given time, such a state of increased engagement is influenced by both affective and cognitive factors. Therefore, pupil dilation reflects the degree rather than the nature of a psychological process. Importantly, in the case of motivation the degree of children's change in pupil dilation is indicative of how much they were engaged by the process of increased tension toward the adult's unfulfilled goal (see also Hepach et al., 2013a). In previous work, changes in pupil dilation have been used to measure infants' and young children's responses to novel events in violation of expectations paradigms (e.g., Gredebäck \& Melinder, 2010; Jackson \& Sirois,
2009). The fact that, in the current Experiment 2, children's pupil dilation increased if the adult was not helped appropriately may be conceived of as a response to an unexpected event. Children remain psychologically engaged if others' needs are not fulfilled appropriately. In addition, the increase in pupil dilation in Experiment 2 suggests that children become engaged as they witness others in need of help. Furthermore, while the results from Experiment 1 show that changes in pupil dilation are linked to motivational strength, the results of Experiment 2 show (a) that this increase is greater when the misplaced objects matter for another person and (b) that this triggered engagement is maintained if the adult is not helped appropriately. Therefore, the present paradigm offers an approach to studying the underlying motives of behavior more generally.

It is important to note that the present experiments only assessed the case of children's instrumental helping for the age group of 2 years. Children's helping occurs in a variety of domains and can be specified according to the type of assistance children provide for others, for example, goods, information, and service (Warneken \& Tomasello, 2009). Likewise, children's helping can also be categorized according to the type of need to which they are responding (e.g., material, emotional, and instrumental; Dunfield et al., 2011). The results from the present experiments address the motivation of children to provide instrumental help. For the emotional helping domain, other studies have shown that children's empathic responding is indicative of their readiness to help another person (Eisenberg \& Miller, 1987; Zahn-Waxler et al., 1992) and that this empathic responding relies to a great extent on children's appreciation of the context in which it occurs (Hepach, Vaish, \& Tomasello, 2013b; Vaish, Carpenter, \& Tomasello, 2009). The adult in the present experiments retained a neutral emotional expression suggesting that the effects on children's anticipatory looking and pupil dilation were not driven purely by an actor's sad emotional expression. This is consistent with previous work demonstrating that children help an adult to an equal degree regardless of whether she displays a sad or a neutral emotional expression (Newton, Goodman, \& Thompson, 2014). Nevertheless, the present findings - and in particular the internal arousal evident when children see others in needmay describe the more general mechanism of children's motivation to help, which could also apply to other domains of prosocial behavior not limited to instrumental helping. Further research is needed 
to identify whether children show similar changes in internal arousal in situations in which others need comfort, information, or resources.

One additional interesting avenue for future research is the systematic investigation of the development of children's motivation to help others. Children's prosocial behavior emerges as early as 12 months for the case of informative pointing (Liszkowski et al., 2008) and at 14 months children begin to help others achieve their instrumental goals (Warneken \& Tomasello, 2007). It is possible that for those younger age groups systematic changes in pupil dilation reveal their motivation to help others, too. On the other hand, children's helping at earlier ages could be motivated by the social engagement of the situation. Another related question is how the results of the present study relate to other domains of social and motor development, for example, whether children would respond with increased pupil dilation to others not being helped appropriately before they themselves have the motor abilities to help others. In this sense, the present experiments provide a starting point for future research on the development of children's intrinsic motivation to help others. As children grow older, their motivation to help others may change and new motives to help, for example, increased concerns for reciprocity, begin to emerge.

In summary, the present results critically extend prior work on the ontogeny of helping behavior by demonstrating that in cases where children help an adult overcome a physical obstacle, they are not simply motivated to see the order of things restored. Rather, children's own internal arousal is mediated by how well another person's need is fulfilled. Importantly, by using a closely matched nonsocial condition we were able to rule out the crucial alternative explanation that children were merely responding to perceptual aspects of the situation, that is, that they simply wanted to see dropped objects returned to their place or see similar objects placed together. Therefore, it appears unlikely that early helping behavior is driven by a concern to "get credit" (Hepach et al., 2012) or driven solely by a desire to complete sequences-both of which have been given as possible explanations nonhuman primate helping behavior (e.g., Köhler, 1925). Instead, the present results lend support to the hypothesis that young children's helping behavior is guided by another person's unfulfilled need. Thus, children genuinely care to see others' well-being maintained or increased.

\section{References}

Beatty, J., \& Lucero-Wagoner, B. (2000). The pupillary system. In J. T. Cacioppo, L. G. Tassinary, \& G. G. Berntson (Eds.), Handbook of psychophysiology (2nd ed., pp. 142162). New York, NY: Cambridge University Press.

Brownell, C., Svetlova, M., \& Nichols, S. (2009). To share or not to share: When do toddlers respond to another's needs? Infancy, 14, 117-130. doi:10.1080/152500008025 69868

Dunfield, K. A., O'Connell, L., Kuhlmeier, V. A., \& Kelley, E. A. (2011). Examining the diversity of prosocial behaviour: Helping, sharing, and comforting in infancy. Infancy, 16, 227-247. doi:10.1111/j.15327078.2010.00041.x

Eisenberg, N., \& Miller, P. A. (1987). The relation of empathy to prosocial and related behaviors. Psychological Bulletin, 101, 91-119. doi:10.1037/0033-2909.101.1.91

Fabes, R., Eisenberg, N., \& Eisenbud, L. (1993). Behavioral and physiological correlates of children's reactions to others in distress. Developmental Psychology, 29, 655. 10.1037/0012-1649.29.4.655

Frank, M. C., Vul, E., \& Saxe, R. (2012). Measuring the development of social attention using free-viewing. Infancy, 17, 355-375.

Gredebäck, G., Johnson, S., \& von Hofsten, C. (2010). Eye tracking in infancy research. Developmental Neuropsychology, 35, 1-9. doi:10.1080/87565640903325758

Gredebäck, G., \& Melinder, A. (2010). Infants' understanding of everyday social interactions: A dual process account. Cognition, 114, 197-206. doi:10.1016/j.cognition.2009.09.004

Hastings, P. D., Zahn-Waxler, C., \& McShane, K. (2006). We are, by nature, moral creatures: Biological bases of concern for others. In M. Killen \& J. G. Smetana (Eds.), Handbook of moral development (pp. 483-516). Mahwah, NJ: Erlbaum.

Hepach, R., Vaish, A., \& Tomasello, M. (2012). Young children are intrinsically motivated to see others helped. Psychological Science, 23, 967-972. doi:10.1177/ 0956797612440571

Hepach, R., Vaish, A., \& Tomasello, M. (2013a). A new look at children's prosocial motivation. Infancy, 18(1), 67-90. doi:10.1111/j.1532-7078.2012.00130.x

Hepach, R., Vaish, A., \& Tomasello, M. (2013b). Young children sympathize less in response to unjustified emotional distress. Developmental Psychology, 49, 11321138. doi:10.1037/a0029501

Hepach, R., \& Westermann, G. (2013). Infants' sensitivity to the congruence of others' emotions and actions. Journal of Experimental Child Psychology, 115(1), 16-29. doi:10.1016/j.jecp.2012.12.013

Jackson, I., \& Sirois, S. (2009). Infant cognition: Going full factorial with pupil dilation. Developmental Science, 12, 670-679. doi:10.1111/j.1467-7687.2008.00805.x

Laeng, B., Sirois, S., \& Gredebäck, G. (2012). Pupillometry a window to the preconscious? Perspectives on Psychological Science, 7(1), 18-27. doi:10.1177/1745691611427305 
Lennon, R., \& Eisenberg, N. (1987). Emotional displays associated with preschoolers' prosocial behavior. Child Development, 58(4), 992-1000.

Liszkowski, U., Carpenter, M., \& Tomasello, M. (2008). Twelve-month-olds communicate helpfully and appropriately for knowledgeable and ignorant partners. Cognition, 108, 732-739. doi:10.1016/j.cognition.2008.06.013

Loewenfeld, I. E. (1958). Mechanisms of reflex dilatation of the pupil. Historical review and experimental analysis. Documenta Ophthalmologica, 12, 185-448. doi:10.1007/BF00913471

Newton, E. K., Goodman, M., \& Thompson, R. A. (2014). Why do some toddlers help a stranger? Origins of individual differences in prosocial behavior. Infancy, 19, 214-226. doi:10.1111/infa.12043

Over, H., \& Carpenter, M. (2009). Eighteen-month-old infants show increased helping following priming with affiliation. Psychological Science, 20, 1189-1193. doi: 10.1111/j.1467-9280.2009.02419.x

R Core Team. (2012). R: A language and environment for statistical computing. Vienna, Austria: R Foundation for Statistical Computing. ISBN 3-900051-07-0. Retrieved from http:/ / www.R-project.org/

Steinhauer, S. R., Condray, R., \& Kasparek, A. (2000). Cognitive modulation of midbrain function: Taskinduced reduction of the pupillary light reflex. International Journal of Psychophysiology, 39(1), 21-39.

Svetlova, M., Nichols, S., \& Brownell, C. A. (2010). Toddlers' prosocial behaviour: From instrumental to empathic to altruistic helping. Child Development, 81, 1814-1827. doi:10.1111/j.1467-8624.2010.01512.x

Vaish, A., Carpenter, M., \& Tomasello, M. (2009). Sympathy through affective perspective taking and its relation to prosocial behavior in toddlers. Developmental Psychology, 45, 534-543. doi:10.1037/a0014322

Warneken, F. (2013). Young children proactively remedy unnoticed accidents. Cognition, 126, 101-108. http:// dx.doi.org/10.1016/j.cognition.2012.09.011

Warneken, F., Hare, B., Melis, A. P., Hanus, D., \& Tomasello, M. (2007). Spontaneous altruism by chimpanzees and young children. PLoS Biology, 5, e184. http:// dx.doi.org/10.1371/journal.pbio.0050184

Warneken, F., \& Tomasello, M. (2006). Altruistic helping in human infants and young chimpanzees. Science, 311, 1301-1303. doi:10.1126/science.1121448

Warneken, F., \& Tomasello, M. (2007). Helping and cooperation at 14 months of age. Infancy, 11, 271-294. doi:10.1111/j.1532-7078.2007.tb00227.x

Warneken, F., \& Tomasello, M. (2008). Extrinsic rewards undermine altruistic tendencies in 20-month-olds. Developmental Psychology, 44, 1785-1788.

Warneken, F., \& Tomasello, M. (2009). Varieties of altruism in children and chimpanzees. Trends in Cognitive Sciences, 13, 397-482. doi:10.1016/j.tics.2009.06.008
Warneken, F., \& Tomasello, M. (2013). Parental presence and encouragement do not influence helping in young children. Infancy, 18, 345-368. doi:10.1111/j.1532-7078. 2012.00120.x

Zahn-Waxler, C., Radke-Yarrow, M., Wagner, E., \& Chapman, M. (1992). Development of concern for others. Developmental Psychology, 28, 126-136. http:// dx.doi.org/10.1037/0012-1649.28.1.126

\section{Supporting Information}

Additional supporting information may be found in the online version of this article at the publisher's website:

Figure S1. An Example Sequence for the Nonsocial Condition Showing the Situation of Stacking Cans

Table S1. Children's Process Pupil Dilation Depending on Their Behavior if They Did Not Help the Adult

Table S2. Results of the Analyses for the Model Investigating Children's Looking at the Objects Prior to the Resolution of the Situation

Table S3. Results of the Analyses for the Model Investigating Children's Looking at the Objects Prior to the Resolution of the Situation Incorporating Both Test Trials

Table S4. Results of the Analyses for the Model Investigating Children's Looking at the Situation Incorporating Both Test Trials

Table S5. Diagnostics for the Model on Children's Change in Pupil Dilation After Witnessing the Objects Drop

Table S6. Results of the Analyses for the Model Investigating Children's Process Measure of Pupil Dilation Prior to the Resolution of the Situation

Table S7. Results of the Analyses for the Model Investigating Children's Process Measure of Pupil Dilation Prior to the Resolution of the Situation Incorporating Both Test Trials

Table S8. Results of the Analyses for the Model Investigating Children's Postmeasure of Pupil Dilation After the Resolution of the Situation

Table S9. Summary of Predictors for the Full Model Including Both Test Trials With the Postchange in Children's Pupil Dilation (After the Resolution of the Situation) as the Dependent Measure

Appendix S1. Additional Experiments 